\title{
Distribusi Normal Multivariat
}

\author{
Husty Serviana Husain ${ }^{1}$
}

\begin{abstract}
Abstrak
Pada pengendalian proses univariat berdasarkan variabel, biasanya digunakan model distribusi normal untuk mengamati kualitas proses dari waktu ke waktu.

Fungsi kepadatan peluangnya (fkp) adalah

$$
f(x)=\frac{1}{\sqrt{2 \pi \sigma^{2}}} \exp -\frac{1}{2}\left(\frac{x-\mu}{\sigma}\right)^{2},-\infty \leq x \leq \infty,
$$

dimana $\mu$ adalah meannya dan $\sigma^{2}$ adalah variansinya. Dalam kenyataannya, seringkali kualitas proses ditentukan oleh lebih dari satu karakteristik yang saling berkorelasi. Dalam hal ini kita bisa saja melakukan pengendalian terhadap masing-masing karakteristik satu-persatu. Akan tetapi hal ini akan menimbulkan banyak sekali kesalahan seperti dikemukakan Montgomery [4]. Oleh karena itu pengendalian proses harus melibatkan semua karakteristik sekaligus. Untuk itu digunakan distribusi normal mulitivariat. Distribusi ini dan beberapa sifatnya merupakan topik bahasan dalam tulisan ini.
\end{abstract}

Kata Kunci: Distribusi Normal Bivariat, Statistical Proces Control .

\section{Pendahuluan}

Pengendalian proses secara statistik (Statistical Proces Control atau disingkat SPC), dibagi ke dalam dua jenis yaitu SPC univariat dan SPC mulitivariat. Dalam SPC univariat hanya satu variabel karakteristik mutu yang dikaji. Kenyataannya, banyak sekali proses yang ditentukan oleh sejumlah karateristik mutu yang terpadu, yang satu sama lainnya berkorelasi. Dalam hal ini, digunakan SPC multivariat. Dengan asumsi bahwa proses memiliki ditribusi normal multivariat. Sehingga tulisan ini akan fokus pada teori distribusi normal multivariat, sebagai distribusi yang digunakan dalam SPC multivariat.

\section{Distribusi Normal Multivariat}

\subsection{Definisi Distribusi Normal Multivariat}

$$
\text { Vektor random yang terdiri atas } p \text { komponen } \quad \vec{X}=\left(X_{1}, X_{2}, \ldots, X_{p}\right)^{t} \quad \text { dikatakan }
$$

berdistribusi normal multivariat dengan vektor mean $\vec{\mu}$ dan matriks variansi-kovariansi $\sum$ yang definit positif, jika fungsi kepadatan peluang bersama $X_{1}, X_{2}, \ldots, X_{p}$ adalah:

$$
f(\vec{x})=\frac{1}{(2 \pi)^{\frac{p}{2}}\left|\sum\right|^{\frac{1}{2}}} \exp \left\{-\frac{1}{2}(\vec{x}-\vec{\mu})^{t} \sum^{-1}(\vec{x}-\vec{\mu})\right\}
$$

\footnotetext{
${ }^{1}$ Universitas Pendidikan Indonesia, email : chery_husty@yahoo.com
} 


\section{Husty Serviana Husain}

dengan $\vec{x}=\left(x_{1}, x_{2}, \ldots, x_{p}\right)^{t}$ di $R^{p}$. Untuk selanjutnya vektor random $\vec{X}$ yang berdistribusi normal $p$-variat tersebut diberi lambang $\vec{X} \sim N_{p}(\vec{\mu}, \Sigma)$. Berikut ini dikemukakan fungsi pembangkit momen dari $\vec{X}$ yang akan digunakan dalam pengkajian distribusi normal multivariat selanjutnya.

\subsection{Teorema 1 Distribusi Normal Multivariat}

Jika $\vec{X} \sim N_{p}(\vec{\mu}, \Sigma)$, maka fungsi pembangkit momen dari $\vec{X}$, ditulis $M_{\vec{X}}(\vec{t})$, dengan $\vec{t}=\left(t_{1}, t_{2}, \ldots, t_{p}\right)^{t}$ adalah $M_{\vec{X}}(\vec{t})=\exp \left(\vec{t} \vec{\mu}+\frac{1}{2} \vec{t} \sum \vec{t}\right)$.

\section{Bukti.}

$$
M_{\vec{X}}(\vec{t})=\exp \left(\vec{t} \vec{\mu}+\frac{1}{2} \vec{t} \sum \vec{t}\right)
$$

Karena $\vec{X} \sim N_{p}(\vec{\mu}, \Sigma)$, maka

$$
\begin{aligned}
& M_{\vec{X}}(\vec{t})=\int_{-\infty}^{\infty} \ldots \int_{-\infty}^{\infty} \frac{1}{(2 \pi)^{\frac{p}{2}}\left|\sum\right|^{\frac{1}{2}}} \exp \left\{\vec{t} \vec{x}-\frac{1}{2}(\vec{x}-\vec{\mu})\right\} d x_{1} d x_{2} \ldots d x_{p} \\
& =\int_{-\infty}^{\infty} \ldots \int_{-\infty}^{\infty} k \cdot \exp \left\{\vec{t} \vec{x}-\frac{1}{2}\left(\vec{x} \Sigma^{-1} \vec{x}-\vec{x}^{t} \Sigma^{-1} \vec{\mu}-\vec{\mu}^{t} \sum^{-1} \vec{x}+\vec{\mu}^{t} \Sigma^{-1} \vec{\mu}\right)+\frac{1}{2}(\vec{\mu} t \vec{t}-\vec{\mu} \vec{t})\right. \\
& \left.;+\left(\vec{t} \sum \vec{t}-\vec{t}^{t} \sum \vec{t}\right)+(\vec{t} \vec{\mu}-\vec{t} \vec{\mu})\right\} d x_{1} d x_{2} \ldots d x_{p} \operatorname{dimana} k=\frac{1}{(2 \pi)^{\frac{p}{2}}\left|\sum\right|^{\frac{1}{2}}} \\
& =\int_{-\infty}^{\infty} \ldots \int_{-\infty}^{\infty} k \cdot \exp \left\{-\frac{1}{2}\left(\vec{x} \Sigma^{-1} \vec{x}-\vec{x}^{t} \sum^{-1} \vec{\mu}-\vec{x} \vec{t}-\vec{\mu}^{t} \sum^{-1} \vec{x}+\vec{\mu}^{t} \sum^{-1} \vec{\mu}\right.\right. \\
& \left.+\vec{\mu} \vec{t}-\vec{t} \vec{x}+\vec{t} \vec{\mu}+\vec{t}^{t} \sum \vec{t}+\frac{1}{2}\left(\vec{t}^{t} \vec{\mu}^{t}+\vec{t}^{t} \sum \vec{t}+\vec{t} \vec{\mu}\right)\right\} d x_{1} d x_{2} \ldots d x_{p} \\
& =\int_{-\infty}^{\infty} \ldots \int_{-\infty}^{\infty} k \cdot \exp \left\{-\frac{1}{2}\left(\vec{x}-\vec{\mu} \sum \vec{t}\right)^{t} \sum^{-1}\left(\vec{x}-\vec{\mu}-\sum \vec{t}\right)+\frac{1}{2}\left(2 \vec{t} \vec{\mu}+\vec{t} \sum \vec{t}\right)\right\} d x_{1} d x_{2} \ldots d x_{p} \\
& =\int_{-\infty}^{\infty} \ldots \int_{-\infty}^{\infty} k \cdot \exp \left\{\frac{1}{2}\left(2 \vec{t} \vec{\mu}+\vec{t}^{t} \sum \vec{t}\right)-\frac{1}{2}\left(\vec{x}-\vec{\mu}-\sum \vec{t}\right)^{t} \Sigma^{-1}\left(\vec{x}-\vec{\mu} \sum \vec{t}\right)\right\} d x_{1} d x_{2} \ldots d x_{p} \\
& =\int_{-\infty}^{\infty} \ldots \int_{-\infty}^{\infty} k \cdot \exp \left(\vec{t} \vec{\mu}+\frac{1}{2} \vec{t}^{t} \sum \vec{t}\right) \exp \left(-\frac{1}{2}\left(\vec{x}-\vec{\mu}-\sum \vec{t}\right)^{t} \sum^{-1}\left(\vec{x}-\vec{\mu} \sum \vec{t}\right)\right) d x_{1} d x_{2} \ldots d x_{p} \\
& =\exp \left(\vec{t} \vec{\mu}+\frac{1}{2} \vec{t}^{t} \sum \vec{t}\right) \int_{-\infty}^{\infty} \ldots \int_{-\infty}^{\infty} \frac{1}{(2 \pi)^{\frac{p}{2}}\left|\sum\right|^{\frac{1}{2}}} \exp \left\{-\frac{1}{2}\left(\vec{x}-\vec{\mu}-\sum \vec{t}\right)^{t} \Sigma^{-1}\left(\vec{x}-\vec{\mu} \sum \vec{t}\right)\right\} d x_{1} d x_{2} \ldots d x_{p}
\end{aligned}
$$




\section{Husty Serviana Husain}

Integral di ruas kanan bernilai 1, sebab integrannya merupakan fungsi kepadatan peluang dari vektor random yang berdistribusi $N_{p}\left(\left(\vec{\mu}+\sum \vec{t}\right), \Sigma\right)$. Oleh karena itu fungsi pembangkit momen dari $\vec{X}$ adalah $M_{\vec{x}}(\vec{t})=\exp \left(\vec{t} \vec{\mu}+\frac{1}{2} \vec{t} \sum \vec{t}\right)$.

\subsection{Teorema 2 Distribusi Normal Multivariat}

Diketahui $\vec{X} \sim N_{p}\left(\vec{\mu}, \sum\right)$ dengan $\sum$ definit positif. Jika $C_{p x p}$ suatu matriks non singular dan $\vec{a}$ suatu vektor skalar di $R^{p}$, maka $\vec{Y}=C(\vec{X}-\vec{a}) \sim N_{p}\left(C(\vec{\mu}-\vec{a}), C \sum C^{t}\right)$.

\section{Bukti.}

Karena $\vec{X} \sim N_{p}(\vec{\mu}, \Sigma)$, maka PDF dari $\vec{X}$ adalah

$$
f(\vec{x})=\frac{1}{(2 \pi)^{\frac{p}{2}} \sqrt{\left|\sum\right|}} \exp \left(-\frac{1}{2}(\vec{x}-\vec{\mu})^{t} \sum^{-1}(\vec{x}-\vec{\mu})\right), \vec{x} \in R^{p}
$$

Karena $C_{p x p}$ non singular, maka $\vec{X}=C^{-1} \vec{Y}+\vec{a}$. Dengan demikian, PDF dari $\vec{Y}$ adalah $g(\vec{y})=f(\vec{x}(\vec{y})) \bmod (J)$, dengan $\bmod (J)$ adalah nilai mutlak dari deterrminan Jacobian transformasi J. Jadi,

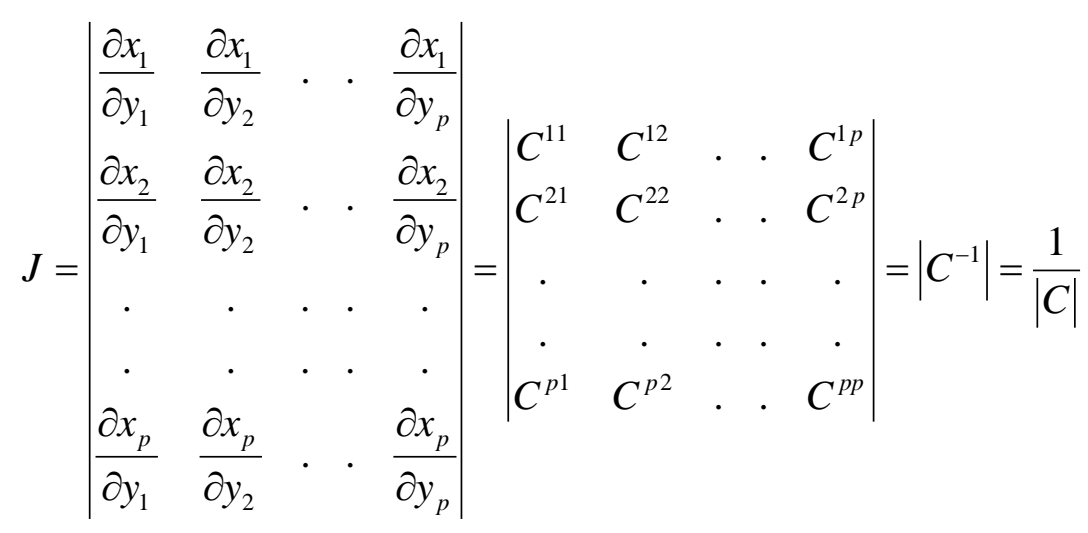

Akibatnya,

$$
\begin{aligned}
& g(\vec{y})=f\left(C^{-1} \vec{y}+a\right) \bmod \left(\frac{1}{|C|}\right) \\
& =\frac{1}{(2 \pi)^{\frac{p}{2}} \sqrt{\left|\sum\right|}} \exp \left(-\frac{1}{2}\left(\left(C^{-1} \vec{y}+\vec{a}\right)-\vec{\mu}\right)^{t} \sum^{-1}\left(C^{-1} \vec{y}+\vec{a}-\vec{\mu}\right)\right) \bmod \left(\frac{1}{|C|}\right)
\end{aligned}
$$




$$
\begin{aligned}
& =\frac{1}{(2 \pi)^{\frac{p}{2}} \sqrt{\Sigma \mid}} \exp \left(-\frac{1}{2}\left(C^{-1} \vec{y}-(\vec{\mu}-\vec{a})\right)^{t} \Sigma^{-1}\left(C^{-1} \vec{y}-(\vec{\mu}-\vec{a})\right)\right) \bmod \left(\frac{1}{|C|}\right) \\
& =\frac{1}{(2 \pi)^{\frac{p}{2}} \sqrt{|\Sigma|}} \exp \left(-\frac{1}{2}\left(C^{-1}(\vec{y}-C(\vec{\mu}-\vec{a}))\right)^{t} \Sigma^{-1} C^{-1}(\vec{y}-C(\vec{\mu}-\vec{a}))\right) \bmod \left(\frac{1}{|C|}\right) \\
& =\frac{1}{(2 \pi)^{\frac{p}{2}} \sqrt{|\Sigma|}} \exp \left(-\frac{1}{2}(\vec{y}-C(\vec{\mu}-\vec{a}))^{t}\left(C^{-1}\right)^{t} \Sigma^{-1} C^{-1}(\vec{y}-C(\vec{\mu}-\vec{a}))\right) \bmod \left(\frac{1}{|C|}\right) \\
& =\frac{1}{(2 \pi)^{\frac{p}{2}} \sqrt{|\Sigma|}} \exp \left(-\frac{1}{2}(\vec{y}-C(\vec{\mu}-\vec{a}))^{t}\left(C \sum C^{t}\right)^{-1}(\vec{y}-C(\vec{\mu}-\vec{a}))\right)\left(\frac{1}{\sqrt{|C||C|}}\right) \\
& =\frac{1}{(2 \pi)^{\frac{p}{2}} \sqrt{|\Sigma|}} \exp \left(-\frac{1}{2}(\vec{y}-C(\vec{\mu}-\vec{a}))^{t}\left(C \sum C^{t}\right)^{-1}(\vec{y}-C(\vec{\mu}-\vec{a}))\right)\left(\frac{1}{\sqrt{|C||C|}}\right) \\
& =\frac{1}{(2 \pi)^{\frac{p}{2}} \sqrt{\left|C \sum C^{t}\right|}} \exp \left(-\frac{1}{2}(\vec{y}-C(\vec{\mu}-\vec{a}))^{t}\left(C \sum C^{t}\right)^{-1}(\vec{y}-C(\vec{\mu}-\vec{a}))\right), \vec{y} \in R^{p}
\end{aligned}
$$

Ini adalah PDF dari vektor acak berdistribusi $N_{p}\left(C(\vec{\mu}-\vec{a}), C \sum C^{t}\right)$. Dengan demikian $\vec{Y}=C(\vec{X}-\vec{a}) \sim N_{p}\left(C(\vec{\mu}-\vec{a}), C \sum C^{t}\right)$.

\subsection{Teorema 3 Distribusi Normal Multivariat}

Misalkan $\vec{X} \sim N_{p}(\vec{\mu}, \Sigma)$ dengan $\sum$ non singulir dan $\vec{X}$ merupakan super posisi $\vec{X}=\left(\begin{array}{l}\vec{X}^{(1)} \\ \vec{X}^{(2)}\end{array}\right)$ dimana $\vec{X}^{(1)}$ dan $\vec{X}^{(2)}$ berturut-turut berdimensi $q$ dan $p-q$. Maka vektor random $\vec{X}^{(1)}$ dan $\vec{X}^{(2)}$ independen jika dan hanya jika matriks kovariansi antara $\vec{X}^{(1)}$ dan $\vec{X}^{(2)}$ adalah $\sum_{12}=0$ (matriks nol).

\section{Bukti.}

Kita tuliskan $\vec{X}^{(1)}=\left(\begin{array}{c}X_{1} \\ X_{2} \\ \cdot \\ \cdot \\ X_{q}\end{array}\right)$ dan $\vec{X}^{(2)}=\left(\begin{array}{c}X_{q+1} \\ X_{q+2} \\ \cdot \\ \cdot \\ X_{p}\end{array}\right)$. 


\section{Husty Serviana Husain}

(i) Misalkan $\vec{X}^{(1)}$ dan $\vec{X}^{(2)}$ independen. Jadi untuk setiap $k=1,2, \ldots, q$ dan setiap $m=$ $1,2, \ldots,(p-q)$, variabel-variabel random $X_{k}$ dan $X_{q+m}$ adalah

$$
\begin{aligned}
\sigma_{k(q+m)} & =E\left[\left(X_{k}-\mu_{k}\right)\left(X_{q+m}-\mu_{q+m}\right)\right] \\
= & E\left[\left(X_{k}-\mu_{k}\right)\right] E\left[\left(X_{q+m}-\mu_{q+m}\right)\right] \\
= & 0
\end{aligned}
$$

Karena ini berlaku untuk setiap $k$ dan $m$, maka

$$
\Sigma_{12}=\left(\begin{array}{ccccc}
\sigma_{1(q+1)} & \sigma_{1(q+2)} & \cdot & \cdot & \sigma_{1 p} \\
\sigma_{2(q+2)} & \sigma_{2(q+2)} & \cdot & \cdot & \sigma_{2 p} \\
\cdot & \cdot & \cdot & \cdot & \cdot \\
\cdot & \cdot & \cdot & \cdot & \cdot \\
\sigma_{q(q+1)} & \sigma_{q(q+2)} & \cdot & \cdot & \sigma_{q p}
\end{array}\right)=\left(\begin{array}{ccccc}
0 & 0 & . & . & 0 \\
0 & 0 & . & . & 0 \\
\cdot & \cdot & \cdot & \cdot & \cdot \\
\cdot & \cdot & \cdot & \cdot & \cdot \\
0 & 0 & . & . & 0
\end{array}\right)
$$

Jadi $\sum_{12}$ adalah matriks nol

(ii) Misalkan $\sum_{12}=0$. Maka matriks variansi-kovariansi dari vektor random $\vec{X}$, yakni $\sum$, dapat dituliskan sebagai berikut.

$$
\Sigma=\left(\begin{array}{ll}
\sum_{11} & \Sigma_{12} \\
\sum_{21} & \sum_{22}
\end{array}\right)=\left(\begin{array}{cc}
\sum_{11} & 0 \\
0 & \sum_{22}
\end{array}\right) \text { dimana } \sum_{21}=\left(\Sigma_{12}\right)^{t}=0
$$

Karena $\Sigma^{-1}=\left(\begin{array}{cc}\Sigma_{11}^{-1} & 0 \\ 0 & \Sigma_{22}^{-1}\end{array}\right)$, maka bentuk kuadrat $Q=(\vec{X}-\vec{\mu})^{t} \Sigma^{-1}(\vec{X}-\vec{\mu})$ dapat pula ditulis sebagai berikut.

$$
\begin{aligned}
Q & =\left(\begin{array}{c}
\vec{x}^{(1)}-\vec{\mu}^{(1)} \\
\vec{x}^{(1)}-\vec{\mu}^{(2)}
\end{array}\right)^{t}\left(\begin{array}{cc}
\sum_{11}^{-1} & 0 \\
0 & \sum_{22}^{-1}
\end{array}\right)\left(\begin{array}{c}
\vec{x}^{(1)}-\vec{\mu}^{(1)} \\
\vec{x}^{(1)}-\vec{\mu}^{(2)}
\end{array}\right) \\
& =\left(\left(\vec{x}^{(1)}-\vec{\mu}^{(1)}\right)^{t}\left(\vec{x}^{(2)}-\vec{\mu}^{(2)}\right)^{t}\right)\left(\begin{array}{cc}
\sum_{11}^{-1} & 0 \\
0 & \sum_{22}^{-1}
\end{array}\right)\left(\begin{array}{c}
\vec{x}^{(1)}-\vec{\mu}^{(1)} \\
\vec{x}^{(1)}-\vec{\mu}^{(2)}
\end{array}\right) \\
& =\left(\vec{x}^{(1)}-\vec{\mu}^{(1)}\right)^{t} \sum_{11}^{-1}\left(\vec{x}^{(1)}-\vec{\mu}^{(1)}\right)+\left(\vec{x}^{(2)}-\vec{\mu}^{(2)}\right)^{t} \sum_{22}^{-1}\left(\vec{x}^{(2)}-\vec{\mu}^{(2)}\right) \\
& =Q_{1}+Q_{2}
\end{aligned}
$$

dimana $Q_{1}$ dan $Q_{2}$ berturut-turut menyatakan suku pertama dan kedua. Selanjutnya karena $|\Sigma|=\left|\Sigma_{11}\right|\left|\Sigma_{22}\right|$, maka f.k.p dari $\vec{X}$ dapat ditulis sebagai berikut. 


$$
\begin{aligned}
f(\vec{x}) & =\frac{1}{(2 \pi)^{\frac{p}{2}} \mid \sum^{\frac{1}{2}}} \exp \left(-\frac{1}{2} Q\right) \\
& =\left\{\frac{1}{(2 \pi)^{\frac{p}{2}}\left|\sum_{11}\right| \frac{1}{2}} \exp \left(-\frac{1}{2} Q_{1}\right)\right\}\left\{\frac{1}{(2 \pi)^{\frac{p-q}{2}}\left|\sum_{22}\right|^{\frac{1}{2}}} \exp \left(-\frac{1}{2} Q_{2}\right)\right\}
\end{aligned}
$$

Sedangkan fungsi yang di dalam tanda kurawal pertama dan kedua berturut-turut merupakan f.k.p dari $X^{(1)}$ dan dari $\vec{X}^{(2)}$. Jadi, $f(\vec{x})=f_{1}\left(\vec{x}^{(1)}\right) f_{2}\left(\vec{x}^{(2)}\right)$ dengan $f_{1}$ dan $f_{2}$ adalah f.k.p marginal dari $X^{(1)}$ dan dari $\vec{X}^{(2)}$. Ini berarti $\vec{X}^{(1)}$ dan $\vec{X}^{(2)}$ independen.

\section{Kesimpulan}

Tulisan ini hanya membahas beberapa teori mengenai distribusi normal multivariat dan pembuktiannya, yang akan banyak digunakan sebagai distribusi dalam pengendalian kualitas proses statistik secara multivariat (SPC multivariat).

\section{Daftar Pustaka}

[1] Anderson T.W., 1958. An Introduction to Multivariate Statistical Analysis. John Wiley \& Son, New York, NY.

[2] Djauhari M.A., 1987. Pengantar Statiska Matematika II. Departemen Pendidikan dan Kebudayaan, Universitas Terbuka.

[3] Hoong R.V., and Craig T.A., 1995. Introduction to Mathematical Statistics, $5^{\text {th }}$ edition. Prentice Hall, Inc., New Jersey.

[4] Montgomery D.C., 2001. Introduction to Statical Qualtiy Control, $4^{\text {th }}$ edition. John Wiley $\&$ Son, New York, NY. 